\title{
Implementing electronic medication management at an Australian teaching hospital
}

edication errors are a continuing and seemingly intractable challenge for our health care system. ${ }^{1}$ The incidence and annual costs are staggering, and most errors are classed as preventable. ${ }^{2}$ All steps of the process of medication management (ordering, dispensing and administration) are subject to error, but prescribing is recognised as the greatest source of error. ${ }^{3}$ Use of an electronic medication management system (eMMS) including computerised physician order entry, a computerised decision support system, and medication administration and pharmacy review components - has been shown to reduce medication errors, although evidence for this is variable. ${ }^{4,5}$ It is acknowledged that the health system has been resistant to the promise of, and investment in, electronic information and communication technologies, but that the momentum for uptake is unstoppable. ${ }^{6}$ We describe our experience of implementing an eMMS in an Australian teaching hospital, in the hope that it will inform similar exercises that are contemplated or in process.

\section{The concept}

In the early 2000s, a number of "home grown" departmental information systems were being developed at St Vincent's Hospital, Sydney, by enthusiastic clinical teams who were concerned about the difficulties associated with paper-based medical record systems. The lack of coordination and governance of these systems constituted a risk to patient safety and stimulated the Chief Information Officer to review commercially available eMMS to add to a number of information technology (IT) modular tools already in place at the hospital, such as the system for pathology results. The Director of Clinical Pharmacology and Toxicology was involved in the national Quality Use of Medicines program, which culminated in the gazettal of the National Medicines Policy in 2000. The policy recognised the inevitability and potential value of electronic health records and electronic prescribing, especially for improving medication safety. ${ }^{7}$

Serendipitously and independently in 2001, two of us (R O D and D J R) had been impressed, from the technical and clinical perspectives, with an Australian eMMS product, MedChart, which the vendors were keen to develop with a teaching hospital. MedChart was selected as the most appropriate product available at the time to implement at St Vincent's Hospital.

\section{Piloting the system}

Pharmacy and nursing staff were initially reserved about the change. Their leaders extrapolated from experience with implementation of other computer-based systems in

\author{
Summary \\ - We describe the implementation of an electronic \\ medication management system (eMMS) in an \\ Australian teaching hospital, to inform future similar \\ exercises. \\ - The success of eMMS implementation depends on: \\ $>$ a positive workplace culture (leadership, teamwork \\ and clinician ownership) \\ $>$ acceptance of the major impact on work practices by \\ all staff \\ $>$ timely system response to user feedback \\ $>$ training and support for clinicians \\ $>$ a usable system \\ $>$ adequate decision support.
}

AM, MD, FRACP,

Director, Clinical Toxicology'

David J Roffe

BSc, BE(Elect), ME(Biomed), Chief Information Officer, Information Technology Service Centre

Katrina L Richardson BPharm, DipHospPharm, Pharmacist, Clinical Information Systems, Department of Pharmacy'

Melissa T Baysari PhD, Research Fellow ${ }^{1,2}$

Nicholas J Brennan MB BS, FRACP. Director, Department of Geriatric Medicine $^{l}$

Sandy Beveridge MB BS, FRACP, Geriatrician and Director of Physician Training, Department of Geriatric Medicine ${ }^{1}$

Teresa Melocco BPharm, GradCertManag, Director of Pharmacy

John Ainge BSc, MB BS, Program Manager, Medication Management ${ }^{3}$

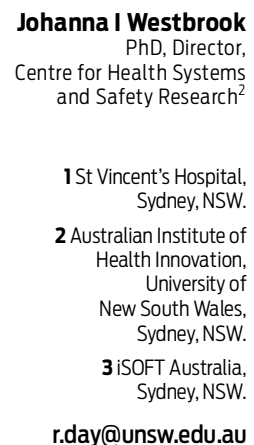

MJA 2011; 195: 498-502 doi: 10.5694/mjall.10451 the hospital and forecast increased workload, insufficient resources and uncertainty of outcomes. In an effort to overcome these initial reservations, individual physicians were identified as "champions" for the change, and their units as potential piloting sites. Two geriatricians (N J B and $\mathrm{SB}$ ) were keen to implement an eMMS, and their service was considered most appropriate for the pilot, as inhospital geriatric patient stays were long, drug therapy was often chronic and multiple medications for geriatric patients was standard.

Most significantly, the executive director of the hospital and the hospital board were enthusiastic. Resources allocated for the project were part of internal annual IT and pharmacy budgets. The buy-in by the executive director and board was based on their agreement that the proposal inherently made sense, it was compatible with the hospital's culture of innovation and goal of reducing adverse medication events, and was a step towards what they believed was an inevitable electronic future.

After 2 years of advocacy and seeking buy-in from stakeholders, a project steering committee was established, comprising members of the medical, nursing, pharmacy, IT and hospital administration departments. A clinical information systems project manager with a clinical background (nursing) was employed by the IT department in early 2004 to coordinate and manage the project. The steering committee met regularly through the period of planning, which took a year, and for the duration of the pilot project.

A commercial arrangement was struck with the vendor and an agreement to develop MedChart with the hospital resulted. Advice was sought from the University of New South Wales Centre for Health Informatics about the likely impact on work practices that might be expected from implementation of a major new IT software package. The advice received was critical to the project and is shown in Box 1. 
1 Advice from the Centre for Health Informatics on clinical implementation of a major new software package

- Ninety-five per cent of successful implementation relies on people, and $5 \%$ on the software package.

- Start with one patient, one doctor, one nurse and one pharmacist.

- Do not add patients to the system until process and system issues are dealt with.

- Training of clinicians is crucial.

The pilot was implemented in April 2005, using one geriatric patient's medication record. After some weeks, another geriatric patient's record was added to the system. One year later, the eMMS was available for all 36 beds in the geriatric ward, with eight portable, wireless-enabled laptop computers fixed to trolleys. These were in addition to the eight fixed desktop computers at clinical work stations in the ward; also clinicians and pharmacists had access to the system from any other computer on the hospital network. The pilot study and roll-out to the entire geriatric ward had been expected to take 3 months. The delays were due to the solving of software and system problems, system enhancements and the changes in work practices identified by users as necessary before further implementation should go ahead. Another significant challenge was maintaining the dual paper and electronic medication systems and the potential for and occurrence of confusion and error. ${ }^{8}$ Placing stickers on paper charts indicating that there was also an electronic chart for that patient was a useful solution.

The vendor responded to our request that the screen of MedChart should replicate the look and feel of the National Inpatient Medication Chart (NIMC) (Box 2); this seemed to be essential to acceptance of MedChart by medical staff. MedChart's patient summary page was also thought to contribute to acceptability. This page includes demographics, allergies and intolerances, the full current medication list, medications on admission and clinician alerts, and so presents a communication and review tool unlike anything on paper.

\section{Implementation}

Despite substantial negative feedback during the pilot (Box 3), the steering committee felt that most issues identified by users had been or would be corrected by the vendor, and that hospital-wide implementation should proceed, so a gradual roll-out followed. The strategic selection of wards for eMMS implementation depended on their casemix and the amenability of the clinical teams. Some clinical teams mounted enthusiastic resistance that was dealt with by responding to their concerns as quickly and effectively as possible. Thus, by May 2006, a second ward (a general surgical ward) was eMMS-enabled, with a much faster implementation time of 11 weeks, assisted by an improved version of MedChart. The steering committee

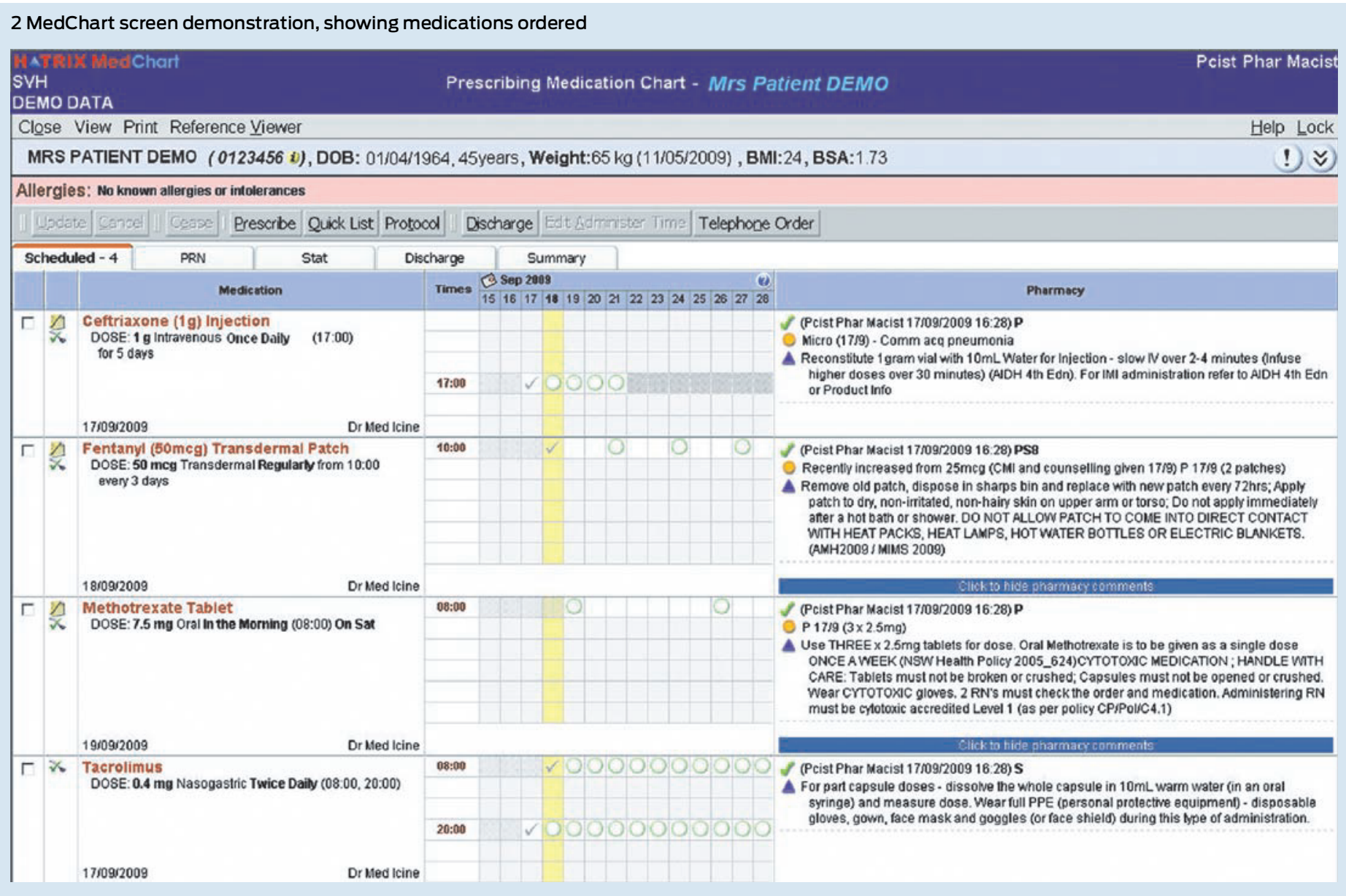




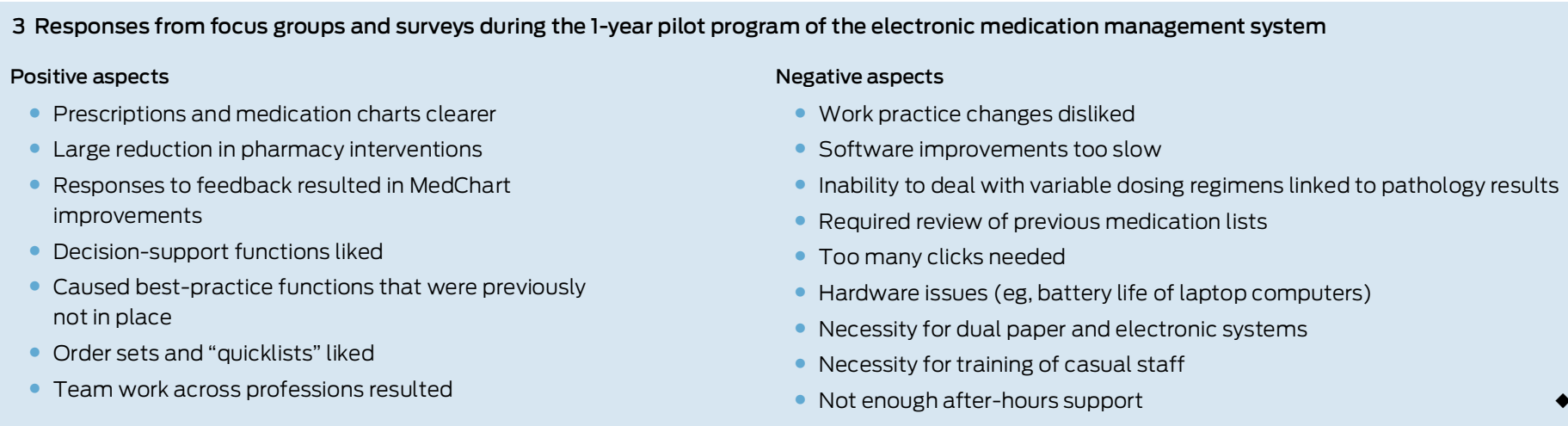

was disbanded and replaced by a representative users' group, and overall governance reverted to a clinical IT committee. The users' group continues to meet monthly and reviews all aspects of the eMMS including "bugs", gripes and suggestions. Some of these result in feedback to the eMMS vendor for attention. Usage rates by ward are reviewed, as are adverse events related to MedChart use. Adverse events are referred to the hospital Drug and Therapeutics Committee and Medication Incident Committee for review.

Paper-based medication ordering still coexists with MedChart, although decreasingly. This is because patients admitted through the emergency department have paper medication charts requiring later transfer to MedChart. The emergency department will begin using MedChart in 2011, and this is expected to almost eliminate the use of paper medication charts.

Significant resistance to implementation of the system by pockets of medical staff, sometimes nurses or pharmacists, has been dealt with in a collegiate manner. One-to-one and group clinical meetings have been attended by users' group members, along with the "champions" from the medical, surgical, IT, pharmacy and nursing departments. These groups hear complaints, reach compromises and propose solutions to problems such as changes to workflow, the software or availability of equipment. For a few clinical groups who initially resisted the change strongly, it took a few months for the benefits to be appreciated. All these exercises were useful, as significant deficiencies in the system were identified and dealt with, and this happened most successfully when medical staff were proactive. For example, complex treatment regimens for immunosuppressed patients (eg, bone marrow transplant patients) are now available as easily charted protocols, and over 77 of these, for a variety of specialties, now are on the eMMS. These order sets can contain over 20 medication orders and are highly regarded by clinical staff.

Despite attempts to involve doctors more deeply in the eMMS roll-out and assessment, their involvement has been limited, compared with that of nurses and pharmacists. However, when specialties have embraced the eMMS (eg, the palliative care, psychiatry, haematology and, most recently, heart and lung transplant units), they have reaped the benefits of "quicklists" and protocols, thus easing their workloads and improving compliance and safety.

\section{Progress to date}

In 2010, every ward of the hospital was using the eMMS, including the high dependency and intensive care units and theatres. The emergency department and day-stay services, with their challenging issues of practice and technology, were to be included in 2011.

Integration and linkage with other IT clinical support systems continue. For example, current medication lists for all inpatients have recently been made available on pathology and imaging results screens to allow quicker clinician review on ward rounds.

More sophisticated electronic decision support (EDS) tools are being incorporated, and are being guided by the Drug and Therapeutics Committee. The technical capability for EDS has always been present, and the view of the steering committee, users' group and drug and therapeutics committee is that a parsimonious approach to decision support is required, identified needs should be proven to be met and "alert fatigue" should be avoided. $^{9}$

\section{Evaluation}

There has been a commitment to evaluation of the eMMS and its implementation since inception of the project. ${ }^{8,10-12}$ This has included not only the feedback from the steering committee, users' group and clinical meetings (eg, medical grand rounds), but also formal evaluations, including focus groups, review of medication incidents, staff satisfaction surveys and pharmacy intervention data. ${ }^{8}$

Focus groups with users on the geriatric ward (medical, nursing and pharmacy staff) at 1, 3 and 8 months of the pilot discussed the arguments for and against eMMS use (Box 3). Actions resulting from these meetings included software enhancements, changes in workflow and practices, improved arrangements for training staff from the agency pool, and lighter trolleys and long-life batteries for the laptop computers.

Small staff satisfaction surveys were conducted before and after the pilot implementation of the eMMS. ${ }^{8}$ There were no differences in the time taken for medication management actions such as prescribing, dispensing and locating medical resources, but staff satisfaction decreased. Before implementation, 100\% of 
4 Lessons learned during pilot implementation and full implementation of the electronic medication management system

- Buy-in and ownership is critical and cannot be rushed or imposed.

- Acceptance of the major impact on work practices by clinicians is vital.

- Transparency of processes is important.

- A lot of communication is necessary using many channels: meetings, in-service training sessions, paper and electronic updates.

- Demonstrable support from the top, leadership from doctors, pharmacists, nurses, information technology employees and the administration department is essential.

- An on-the-ground, at-the-ready, immediate problem-solving team is important. Solving a problem quickly leads to increased user acceptance and knowledge.

- It is important to train users in varied aspects of the system - for example, pharmacists trained in the basics of prescribing and administration functions can act as a resource on the wards to answer simple medical and nursing queries.

- Recognise the value of "champions" for change (formal and informal) and ward-level leadership.

- A controlled roll-out builds confidence and identifies problems.

- Use agreed timelines and milestones to avoid frustrations.

- Revise roll-out and implementation plans if necessary in response to experience.

- Interpretation of feedback and evaluation is important to dissect problems related to software, process and practice.

- Deal with clinician expectations and response time of technical and software fixes.

- Provide constant evaluation and feedback to users.

- Foster a productive relationship with the software vendor to adjust the product to local needs and specifications.

- Local adaptability of the system is needed for local clinical and administrative needs.

- Give limited decision support and careful introduction initially to avoid user frustration. For example, our drug interactions database is not yet activated.

respondents felt that eMMS would be beneficial to patient care, but after implementation, this dropped to $38 \%$. There was also a decrease in satisfaction with the functionality of the system, with 12 of 19 participants dissatisfied or very dissatisfied with the electronic system. No users were dissatisfied with the paper-based system before eMMS implementation.

Despite this decline in satisfaction following the pilot implementation of the eMMS, pharmacy interventions to adjust medication regimens in the interests of safety and compliance with hospital policies dropped by $55 \%$ after implementation. Evidence of reduction in medication errors has been obtained at various stages of the roll-out. For example, an audit of medication charts by clinical pharmacists before and after eMMS implementation on one ward showed a significant decrease in rates of prescribing error. Following eMMS implementation, error rates decreased by over $50 \%$ (from 22.5 per 100 patient-days [95\% CI, 19.9-24.1] to 9.0 per 100 patient-days [95\% CI, 8.8-9.2]). ${ }^{10}$

Training investment is critical, and more is always needed. We have had a clinical information systems project manager, a pharmacist dedicated to the project, large input from the clinical pharmacology registrar, and during the pilot we hired a dedicated clinical information systems trainer, a position that has been retained. It has remained a challenge to support all staff, especially the rotating and casual nursing workforce. Many innovations have been introduced, including identifying "powerusers" (eMMS users with a high degree of competency) as volunteer educators, more mandatory training sessions and e-learning modules. In the early stages of the project, more assistance for training would have been beneficial. We now use after-hours nurse managers, nurse educators, and on-call pharmacy and IT staff to good effect in training roles.

\section{Conclusions}

The eMMS is a work in progress. We are excited about the gains in safety and efficiency that are possible with targeted and sophisticated EDS, but at the same time are cautious about causing frustration and irritation to clinicians by excessive and unwanted intrusions into their time. ${ }^{12}$ We are also looking forward to greater integration with other information systems, such as the pathology service, so that drugs requiring variable dosing regimens linked to pathology data (eg, gentamicin, warfarin and insulin) can be prescribed efficiently and safely. We are enthusiastic about more innovative education and training, customised to the needs of individual clinicians and their experience and seniority. We believe these approaches will enhance staff satisfaction. We have barely explored the large potential for individual feedback to prescribers to enhance their performance and patient safety. We also expect to capitalise on our ability to measure our performance as an organisation using the reporting module capabilities of the MedChart system.

We need to continue to invest in hardware and software to optimise performance. We also acknowledge the challenges of keeping up with changes in software and technologies, and meeting clinical needs, and we regard this as a necessary cost of operations. We consider that eMMS implementation and results have been a success for our hospital, and we have learned a lot about what has contributed to that success (Box 4).

Successful implementation of an eMMS with EDS depends on many factors, most importantly:

- a positive workplace culture with leadership at all levels, teamwork and clinician ownership 
- an acceptance of the major impact on work practices by all staff

- the ability to modify the system in a timely manner in response to user feedback

- appropriate training and support for clinicians

- good usability (does it look and feel right and work well?)

- neither too much or too little decision support.

Acknowledgements: We thank Kerry Stubbs, Jonathan Anderson, Maureen Heywood, Garry Nichols, Darren Roberts, Jo Fowler, Silvia Fazekas, the late Tony Jones, Enrico Coiera and Dennis Armstrong for their support, advice, leadership and important contributions to the project.

Competing interests: John Ainge is employed by iSOFT, the vendors of MedChart. Provenance: Not commissioned; externally peer reviewed.

1 Roughead EE, Semple SJ. Medication safety in acute care in Australia: where are we now? Part 1: a review of the extent and causes of medication problems 2002-2008. Aust New Zealand Health Policy 2009; 6: 18

2 Dartnell JG, Anderson RP, Chohan V, et al. Hospitalisation for adverse events related to drug therapy: incidence, avoidability and costs. Med J Aust 1996; 164: 659-662.

3 Bates DW, Cullen DJ, Laird N, et al. Incidence of adverse drug events and potential adverse drug events. Implications for prevention. ADE Prevention Study Group. JAMA 1995; 274: 29-34.

4 Reckmann MH, Westbrook Jl, Koh V, et al. Does computerized provider order entry reduce prescribing errors for hospital inpatients? A systematic review. Am Med Inform Assoc 2009; 16: 613-623.

5 Australian Commission on Safety and Quality in Health Care. Electronic medication management systems: a guide to safe implementation. Sydney: ACSQHC, 2011. http://www.health.gov.au/internet/safety/publishing.nsf/ Content/com-pubs_emms-safe-implementation (accessed Sep 2011).

6 Westbrook Jl, Braithwaite J. Will information and communication technology disrupt the health system and deliver on its promise? Med J Aust 2010; 193: 399-400.

7 Australian Government Department of Health and Ageing. National Medicines Policy 2000. Canberra: DoHA, 1999. http://www.health.gov.au/internet/main/ publishing.nsf/Content/nmp-objectives-policy.htm (accessed Aug 2011).

8 Fowler J, Hyde G. Case study: implementing an electronic medication management system. J Aust Assoc Qual Health Care 2007; 17: 5-9.

9 Van Der Sijs H, Aarts J, Vulto A, Berg M. Overriding of drug safety alerts in computerized physician order entry. J Am Med Inform Assoc 2006; 13: 138-147.

10 Westbrook JI, Lo C, Reckmann MH, et al. The effectiveness of an electronic medication management system to reduce prescribing errors in hospital. In: Hansen DP, Schaper L, Rowlands D, editors. Informing the business of healthcare. Proceedings of the 18th Annual Health Informatics Conference; 2010 Aug 24-26; Melbourne. Melbourne: Health Informatics Society of Australia, 2010: 96-100. http://search.informit.com.au/ documentSummary:dn=430012692310760;res=IELHEA (accessed Aug 2011).

11 Magrabi F, Li SY, Day RO, Coiera E. Errors and electronic prescribing: a controlled laboratory study to examine task complexity and interruption effects. J Am Med Inform Assoc 2010; 17: 575-583.

12 Baysari MT, Westbrook Jl, Richardson KL, Day RO. The influence of computerized decision support on prescribing during ward-rounds: are the decision-makers targeted? J Am Med Inform Assoc 2011. Epub Jun 14. doi:10.1136/amiajnl-2011-000135.

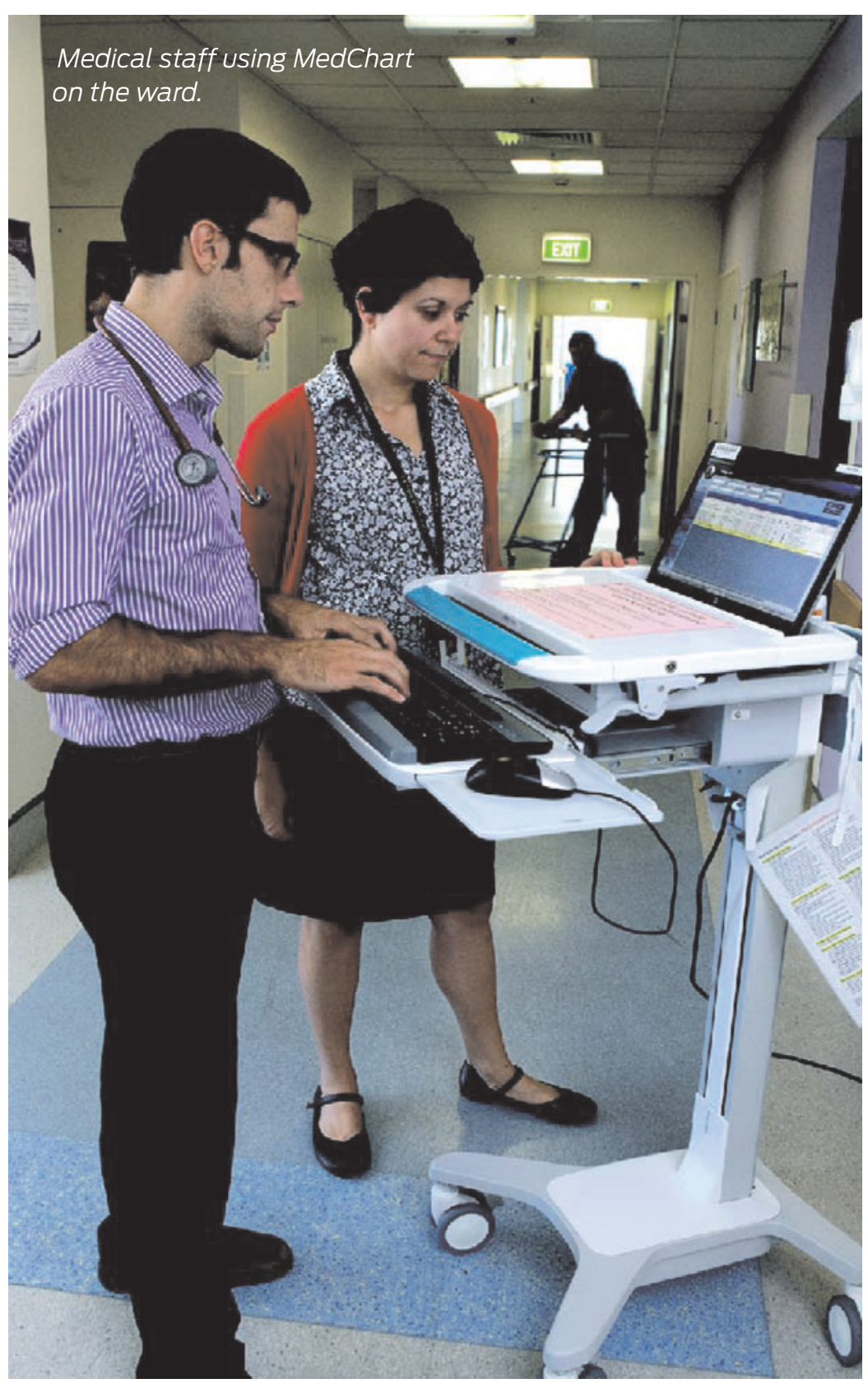

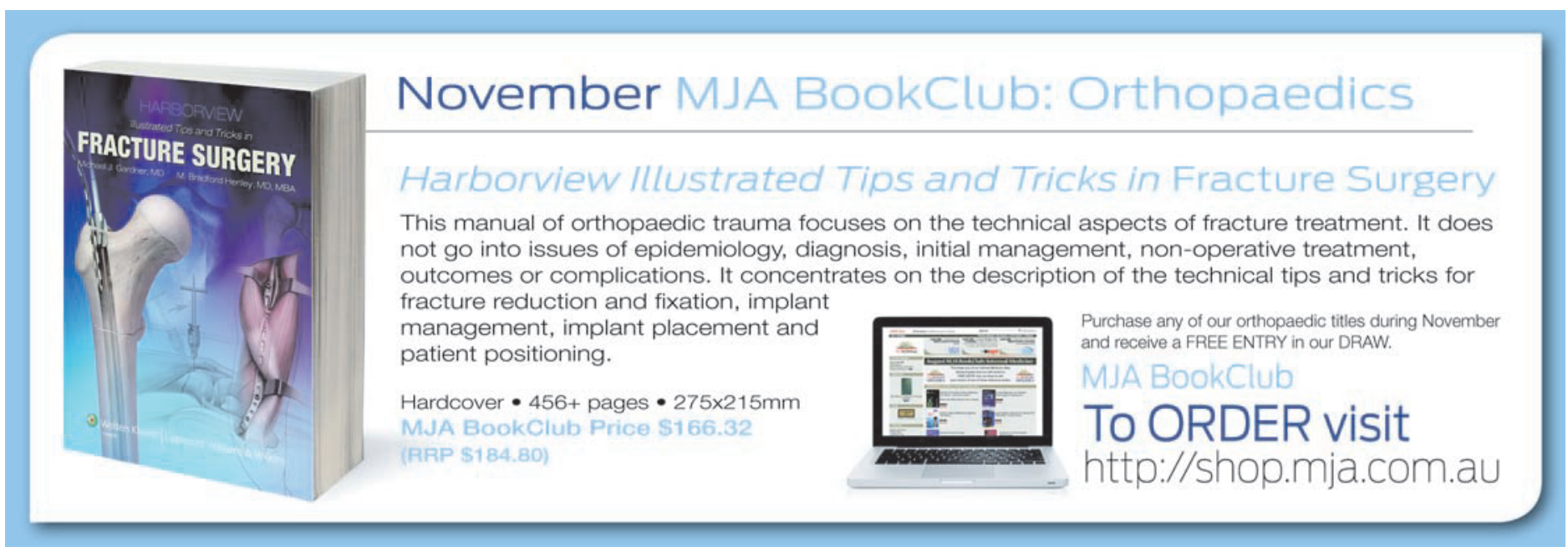

